\title{
Estudio taxonómico de Chilenoperla puerilis (Insecta, Plecoptera), y su asignación a Ericiataperla nueva combinación
}

\section{Taxonomic Study of Chilenoperla puerilis (Insecta, Plecoptera), and her assignment to Ericiataperla new combination}

\author{
Alejandro Vera S.*1 \\ ${ }^{1}$ Departamento de Biología, Facultad de Ciencias Básicas, Universidad Metropolitana de Ciencias de la Educación, Av. José \\ Pedro Alessandri 774, Ñuñoa, Santiago, Chile. \\ *E-mail alveras2@gmail.com
}

\begin{abstract}
RESUMEN
Se realiza un estudio taxonómico de $C$. puerilis. Se redescribe la ninfa y describen los adultos de ambos sexos. Se identifican 17 rasgos en oposición con las restantes especies de Chilenoperla seis en adultos y once en ninfas. Estos resultados apoyan una nueva combinación nomenclatural para la especie dentro de un nuevo género nombrado Ericiataperla. El caso estudiado muestra la relevancia de asociar correctamente los estados de desarrollo en insectos acuáticos, para así asignarles atributos ecológicos y estimar la diversidad de los taxa involucrados.
\end{abstract}

Palabras Clave: Gripopterygidae, insectos acuáticos, morfología, ninfas.

\begin{abstract}
I perform a taxonomic study for C. puerilis. From the description of male and female adults, and redescription of the nymph, six adult and eleven immature traits were identified in opposition to the remaining species of Chilenoperla. With these results, I propose a new genus, Ericiataperla, at which I have assigned the species in question under a new combination. The case study shows the importance of correctly associate the developmental stages in aquatic insects to assign ecological attributes and estimate the diversity of the taxa involved.
\end{abstract}

KEYwords: Aquatic insects, Gripopterygidae, morphology, nymphs.

\section{INTRODUCCIÓN}

El género Chilenoperla Illies 1963, fue establecido junto a la descripción de tres especies, dos de ellas sobre la base de imagos adultos (C. semitincta y C. beschi) y una con su ninfa y el farato macho parcialmente formado que contenía (C. puerilis). Posteriormente Nelson (1973) describe $C$. illiesi con imagos macho y discute la morfología genital en un análisis cladístico de las cuatro especies descritas. Tras 35 años sin contribuciones Vera (2008) describe a $C$. elongata y más tarde a C. puelche Vera 2012, ambas especies con imagos macho, hembras y sus ninfas. Sin embargo la morfología ninfal resultó distar mucho de la previamente descrita para C. puerilis y por el contrario se asemeja a la de Pelurgoperla personata Illies 1963. A diferencia de $C$. puerilis las ninfas de C. elongata, C. puelche y P. personata presentan camuflaje por enmascaramiento y adaptaciones asociadas, como son la falta de esclerosis en la mayor parte de su cuerpo, el desarrollo de setas cintiformes, largas y flexibles, el estrangulamiento de los segmentos abdominales, entre otros. Por otra parte $C$. puerilis presenta una ninfa conspicua y distinguible de cualquier otro género conocido para América del Sur, donde el rasgo morfológico más relevante es el particular tipo de setas con aspecto de púas y su distribución en los márgenes de pro, meso, metanoto y segmentos abdominales, además del labio y maxila. Por otra parte en los imagos la porción genital mejor conservada del farato es el epiprocto, cuyo ápice ampliamente redondeado difiere de todas las restantes especies del género, donde su extremo apical es estrecho. Este contraste en la morfología de ninfas e imagos motivo la hipótesis de que $C$. puerilis en realidad correspondiera a otra taxa y no a Chilenoperla, Sin embargo el desconocimiento de los imagos de C. puerilis ha impedido realizar un estudio más acabado.

Desde que Chilenoperla fuera descrito y dada la relevancia de la identificación de estados inmaduros en los estudios limnológicos, la ninfa de $C$. puerilis ha sido incorporada en 
una serie de herramientas de identificación (Froehlich 2009, Stark et al. 2009, McLellan \& Zwick 2007) así como en estudios donde se desprenden para ellas diversos atributos ecológicos (Miserendino 2000, 2001, 2006; Miserendino \& Pizzolón 2000, 2003, Velásquez \& Miserendino 2003a, 2003b, Figueroa et al. 2003), estos registros han identificado a la especie desde su localidad tipo en Chile (Llanquihue, Peulla) a Argentina Chubut, consignándose así en el catálogo más recientemente publicado (Froehlich 2010).

En este estudio fue posible asociar los imagos machos y hembras con las ninfas de $C$. puerilis mediante emergencia de imagos in situ en una localidad de Chile, Región de Aisén. Este material permite conocer por primera vez los imagos de ambos sexos, y reconocer en la morfología imaginal una serie de rasgos asociados a la genitalia, tórax y venación alar, estos discrepan con las restantes especies de Chilenoperla y permiten proponer una nueva combinación genérica para la especie C. puerilis, el que se nombra como Ericiataperla. Se presenta una redescripción de la ninfa, la descripción de los imagos de ambos sexos y un mapa de los registros del material examinado, extendiendo su presencia a Tierra del Fuego.

\section{MATERIALES Y MÉTODOS}

Los adultos fueron colectados mediante sacudida del follaje a orillas del curso de agua, las ninfas con red acuática rectangular y la asociación de ambos estados fue mediante colecta in situ de especímenes emergiendo. Todos los especímenes fueron conservados en alcohol de 70\%, Las disecciones y mediciones corporales fueron realizadas con lupa estereoscópica Nikon (oculares 10X, objetivos zoom $0,7 \mathrm{X}$ a $3 \mathrm{X}$ ), con reglilla incorporada al ocular. Las figuras se elaboraron con cámara clara en un microscopio Leitz Dialux 22 Bauchamp-loop y refinados posteriormente, las fotografías con cámara digital incorporada a ambos equipos ópticos. Los especímenes conservados en preparaciones microscópicas fueron tratados con $\mathrm{KOH} 10 \%$ sin calentar por $12 \mathrm{hrs,} \mathrm{montados} \mathrm{en} \mathrm{solución}$ Doetschman (Camousseight \& Fontaine 1990), previamente las alas se desprendieron y montaron sin pasar por $\mathrm{KOH}$. Las fotografias SEM fueron tomadas en un microscopio electrónico de barrido HITACHI TM3000. La nomenclatura de las alas sigue la propuesta de Béthoux (2005).

Material examinado: Tipos examinados: Holotipo: farato y ninfa ${ }^{\lambda}$, Chile: X Región, Prov. Llanquihue, Laguna Margarita, Peulla, 1100msnm, 18-I-1954, leg. Brundin, en colección Illies. Paratipos: 10 ninfas con los mismos datos. Otro material examinado: CHILE: X Región, P.N. Vicente Pérez Rosales, Casa Pangue, Límite 16-III-1974, col J. C. Ortiz (1 $\overbrace{}^{\Uparrow})$; XI Región, Cascada estero Co. Paloma, cerca del lago Elizalde 31-X-2008, col. A. Vera (4⿳⺈, 10 ninfas, 2 exuvias); 15-II-2007, col A. Vera (2 exuvias); XII Región, Cañadón Doña Rosa, Glacial Tyndall, 17-I-2003, col. A. Zuñiga (1 $\hat{\gamma}, 1$ 우); Parque Nacional Torres del Paine 10 II 2006 Leg. Thibault (5 ninfas); XI Región, Coyhaique, Río Manso, 23-II-2001, col M. Guerrero (1 )); XI Región, PN. Laguna San Rafael, Valle de Soler, 31-I-1999, col A. Vera (1ㅇ); XII Región, Tierra del Fuego, Pto. Williams, Parque Omora, Karukinka 30-X-2006, col C. Anderson (2 ninfas). ARGENTINA: Prov. Neuquén, 10:Chapelco-Techos, 1400 MSNM, 14-XI-1982, col M. y P. Gentili, colección IADIZA (1今); Prov. Río Negro Lago Nahuel-Huapi, col R. Albariño (2 ninfas); Prov. Chubut, Estero La Hoya 21-IX-2004, col Hollmann (5 ninfas), Robalo 18-II-2003 (1 ninfa).

\section{RESULTADOS}

Ericiataperla $n$. gen. Vera

(Figs. 1 a-f, 2 a-f, 3c-d, 4 a-d, 5 a-e, 6)

DESCRIPCIÓN. Imagos de color negro a pardo, con alas ahumadas de color pardo y bandas blancas variables en torno a las venas transversales. Ambos pares de alas sin o con escasas venas transversales-interrumpidas, horquilla de RS sin vena transversal (ausencia de celda subapical), en su lugar una mancha blanca en la lámina (Figs. 1 a y b). Pronoto transversal de lados paralelos y vértices angulosos en machos (Fig. 1d) redondos en hembras (Fig.1e), superficie central convexa, márgenes posteriores de meso y metanoto cóncavo. Genitalia masculina con epiprocto de ápice amplio y forma de "pala", dorsalmente con cuatro a cinco pares de dientes transversales de ápice redondeado (Figs. 3c y d); paraproctos fusiformes (Fig. 2e), con el cuarto apical doblado por sobre el esclerito central del tergo X (Fig. 2 a y b). Ninfas: pardas de aspecto cilíndrico y ligeramente aplanadas dorsoventralmente; con antenas y cercos filiformes (Fig. 4 a) con cerdas cortas y dispuestas paralelas al artejo; pronoto cuadrado, meso y metanoto de margen posterior convexo y recto respectivamente, con pterotecas desarrolladas, segmentos abdominales IV a $\mathrm{X}$ como anillos, de lados rectos, último segmento en vista dorsal con su margen distal ampliamente redondeado; patas delgadas y con garras extendidas (Fig. 5 a). Armadura bucal con mandíbulas planas con dientes incisivos y molares de margen filoso, mandíbula izquierda con 4 incisivos (Fig. 5b); maxilas con palpo de segmentos largos y cerdas erizadas, su longitud supera 2,5veces la lacinia (Fig. 5c); lacinia con dientes y cerdas agudas, el margen dorsal con una lámina que forma una ligera concavidad; labio con palpos de segmento distal dilatado medialmente. Cerdas conspicuas, planas muy esclerosadas y con forma de "púas" en el margen del pronoto, meso y metanoto, pterotecas, margen posterior de todos los segmentos abdominales (Figs. $4 \mathrm{a}, \mathrm{b}$ y c), patas con cerdas cortas. 
ETIMologíA. Ericiataperla $=$ palabra compuesta derivada del latín ericiatus $=$ erizado, que se obtiene de adjetivar ericius $=$ erizo y perla $=$ nombre propio femenino, referido habitualmente a los perlarios. El nombre genérico hace referencia al tipo de cerdas pequeñas, con aspecto de "púas" erizadas que son muy evidentes en los márgenes del pro meso y metanoto, segmentos abdominales y armadura bucal de las ninfas.
Ericiataperla puerilis (Illies, 1963) nov. comb.

Chilenoperla puerilis Illies, 1963:226.

DESCRIPCIÓN DE IMAGOS

Imagos Macho (Figs. 1 a, d; 2 a-f; 3c y d):

Dimensiones, longitudes en $\mathrm{mm}$ (promedios $\pm \mathrm{DE}$ ): cuerpo $8,16 \pm 0,74$; antena $9,16 \pm 1,07$; cerco $0.73 \pm 0,07$; ala anterior $11,16 \pm 1,22$.

Machos: coloración corporal mayoritariamente negra; Cabeza solo con regiones anaranjadas en el clípeo, sutura
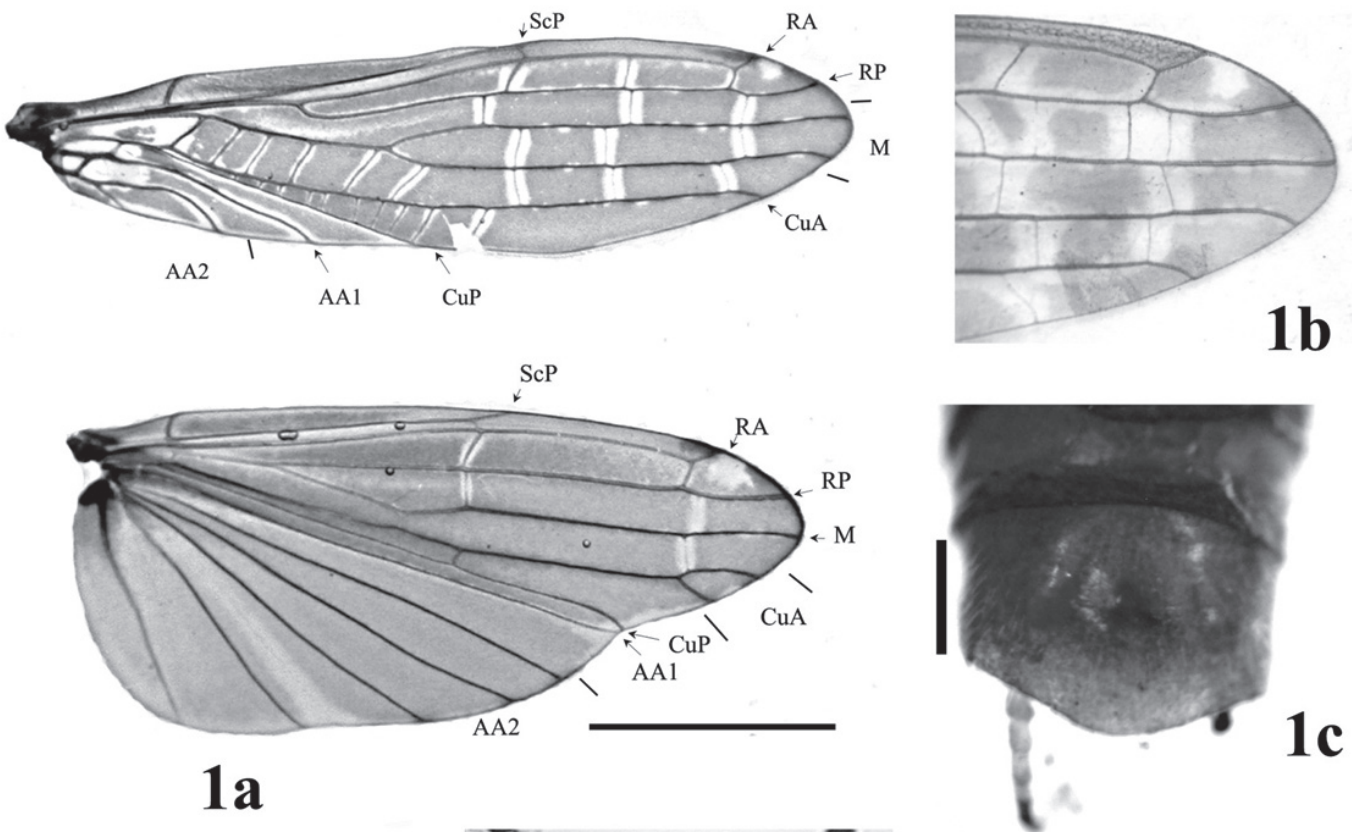

$1 \mathbf{a}$

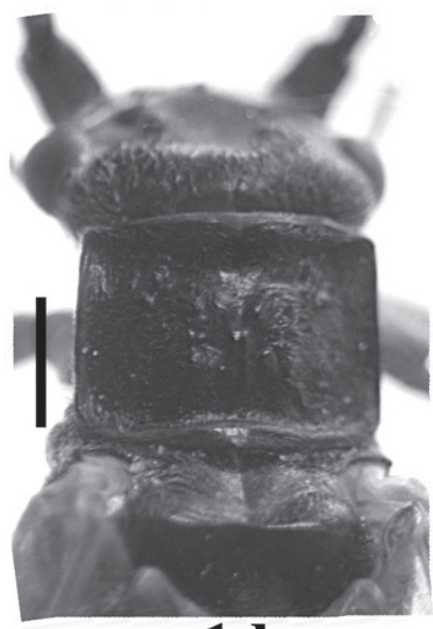

$1 d$
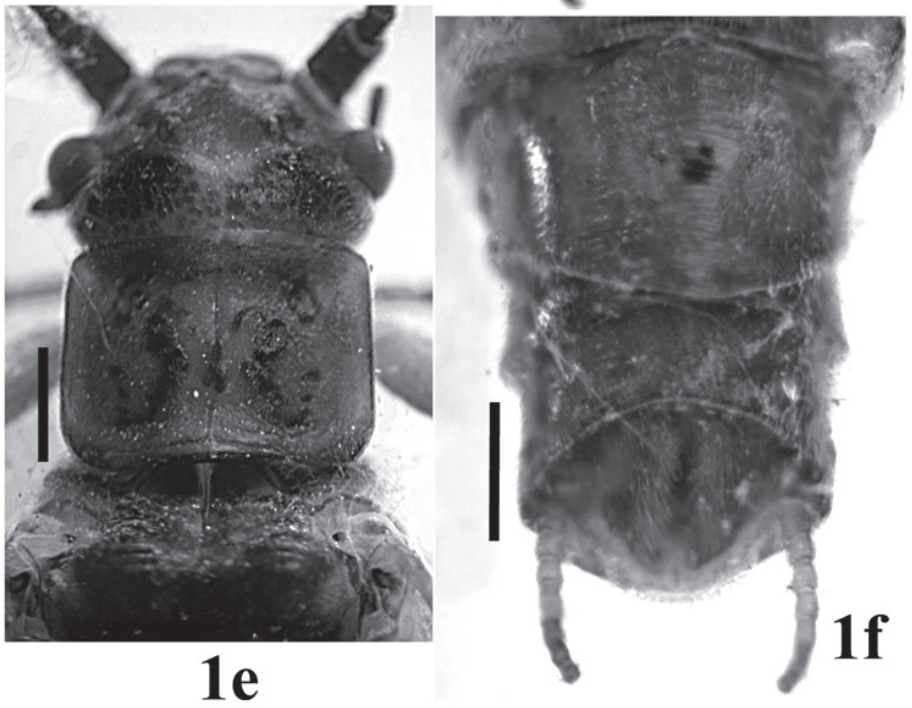

Figuras 1a-f. Ericiataperla puerilis Nov. comb. adultos: a) Alas $\widehat{\partial}$, escala 4,0mm; b) Ala anterior $q$, ápice; c) Abdomen $\odot$, dorsal, escala

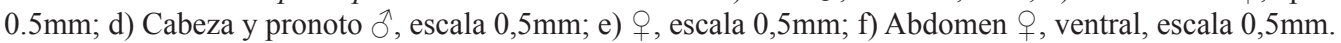

Figures 1a-f. Ericiataperla puerilis Nov. comb. adults: a) Wings, scale 4.0mm; b) Apex wings + ; c) Abdomen + , dorsal, scale 0.5mm; d) Head and pronotum $\widehat{\partial}$, scale $0.5 \mathrm{~mm}$; e) Female, scale $0.5 \mathrm{~mm}$; f) Abdomen + , ventral, scale $0.5 \mathrm{~mm}$. 
coronal y piezas de la armadura bucal, con palpos labiales y maxilares grises; Patas con tibias y tarsos grises; Abdomen con segmento I al VIII pardo oscuros, con región pleural libre no esclerosada; Segmentos IX y X amarillentos.

Cabeza con ojos negros; ocelos cristalinos, con una profunda hendidura entre los ocelos laterales y el punto de bifurcación de la sutura coronal, impresiones postantenales oscuras de forma subcircular; antenas con primer segmento del flagelo corto, semejante a la suma de los dos siguientes.
Tórax: pronoto (Fig. 1d) transversal, margen anterior y posterior recto de lados paralelos, vértices anteriores y posteriores de ángulos rectos; meso y metanoto con margen posterior convexo; Patas largas y delgadas. Ambos pares de alas (Fig. 1a) con lámina pardo oscuro, pterostigma rojizo, venas transversales rodeadas de una mancha blanca, con escasas o ninguna vena interrumpida, RS con horquilla, pero sin celda subapical, presenta una mancha blanca entre las ramas de $\mathrm{RS}$.

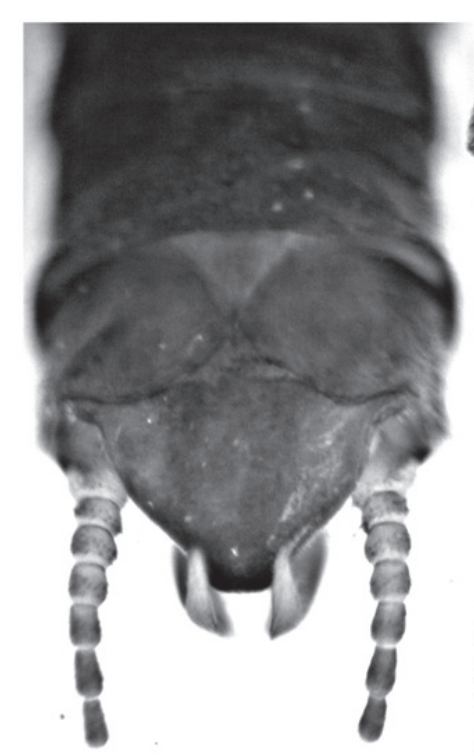

2a

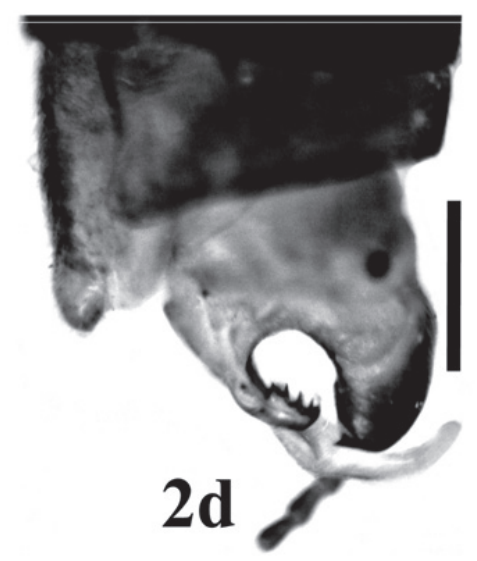

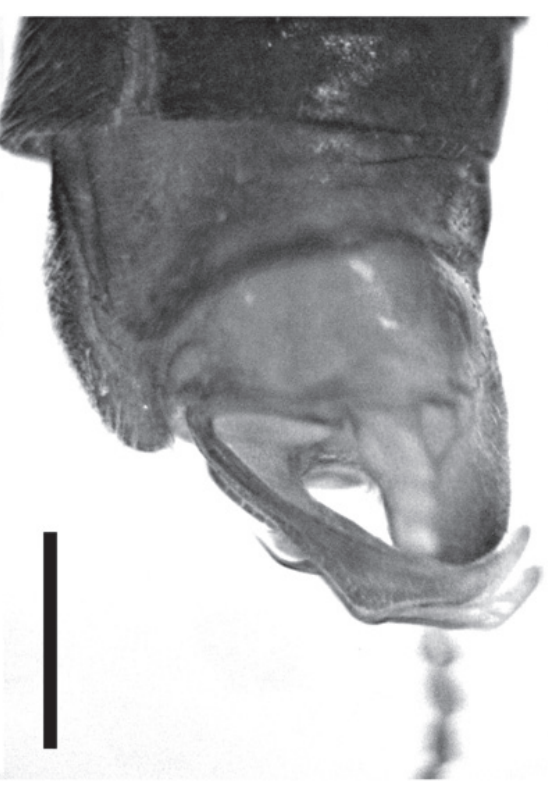

2b

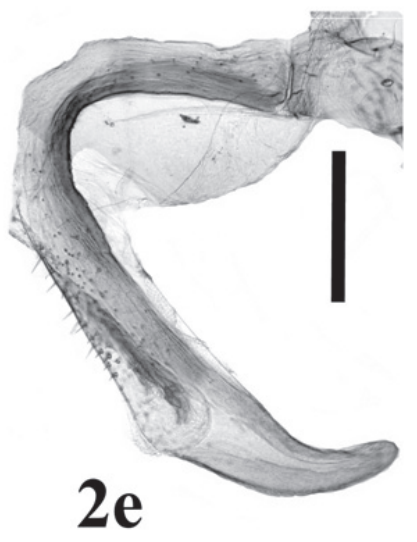

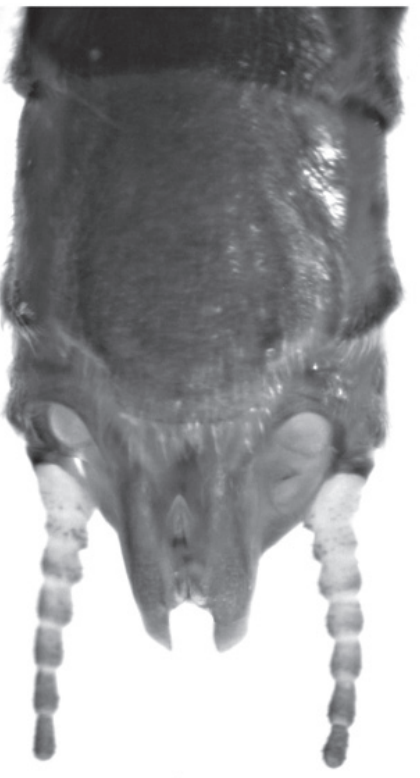

2c

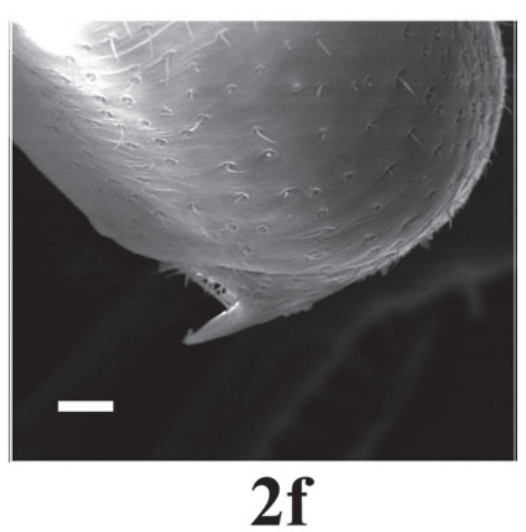

Figuras 2a-f. Ericiataperla puerilis Nov. Comb. adulto $\hat{0}$ : a-d) Abdomen dorsal, lateral, ventral y lateral sin paraprocto, escala 0,5mm; e) Paraprocto, escala $0,25 \mathrm{~mm}$; f) Esclerito posterior con SEM escala $20 \mu \mathrm{m}$.

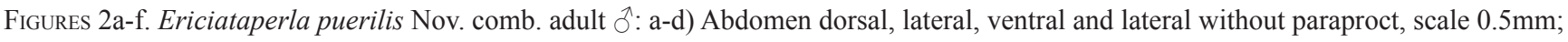
e) Paraproct, scale $0.25 \mathrm{~mm}$; f) Posterior sclerite with SEM, scale $20 \mu \mathrm{m}$. 
Abdomen: segmento IX esclerosado, la región esternal esta unida mediante una membrana lateral; esterno convexo, de forma rectangular, de margen posterior prominente y redondeado (Fig. 2c). Segmento $X$ con escleritos anteriores separados, esclerosados y convergentes hacia la línea media dorsal (Fig. 2 a), con un cayo poco evidente en su margen posterior; esclerito central unido a los laterales (Fig. 2 a), dorsalmente plano, de margen anterior recto, aguzado por sobre los cercos, margen posterior prominente, de ápice truncado y redondeado; esclerito posterior ubicado por debajo del ápice del esclerito central (Fig. 2d y f), libre, de color negro, con forma de una corta lámina transversal, de ápice agudo; epiprocto con forma de "pala", de superficie dorsal plana, sobresaliendo 4 a 5 dientes en los márgenes, estos dientes se disponen como laminas transversales de base amplia y forma triangular (Figs. 3c y d), en vista lateral se ven como delgadas y largas láminas, ápice del epiprocto amplio y redondeado, puede hendirse medialmente, dando el aspecto de bilobulado o asimétrico; tras el diente posterior se estrecha violentamente en una corta lamina, en vista lateral sobresale una larga quilla, plana, angosta y flectada hacia posterior, bajo el epiprocto no se presentan abultamientos membranosos (Fig. 2d); Paraproctos largos, fusiformes (Fig. 2e), de ápice ancho y redondeado, con

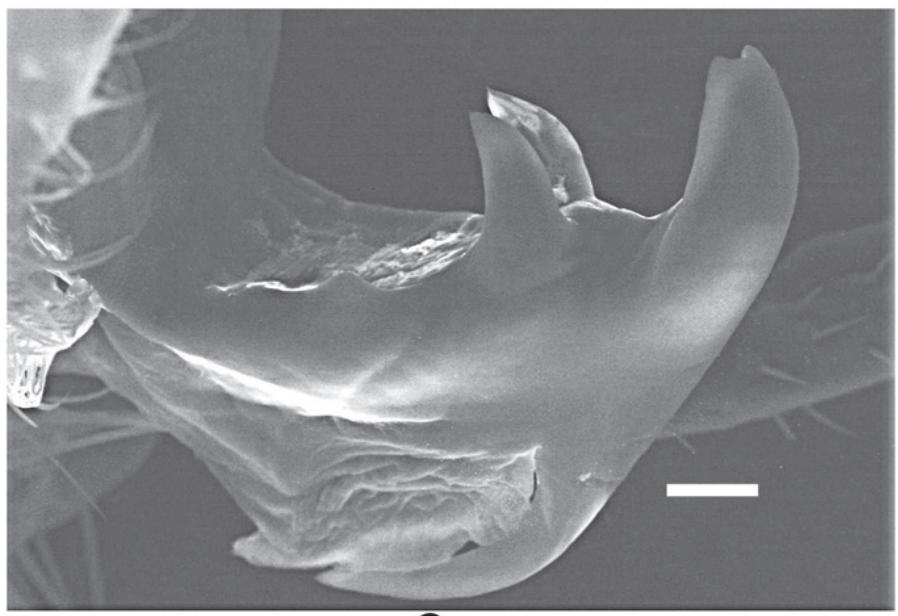

$3 \mathbf{a}$

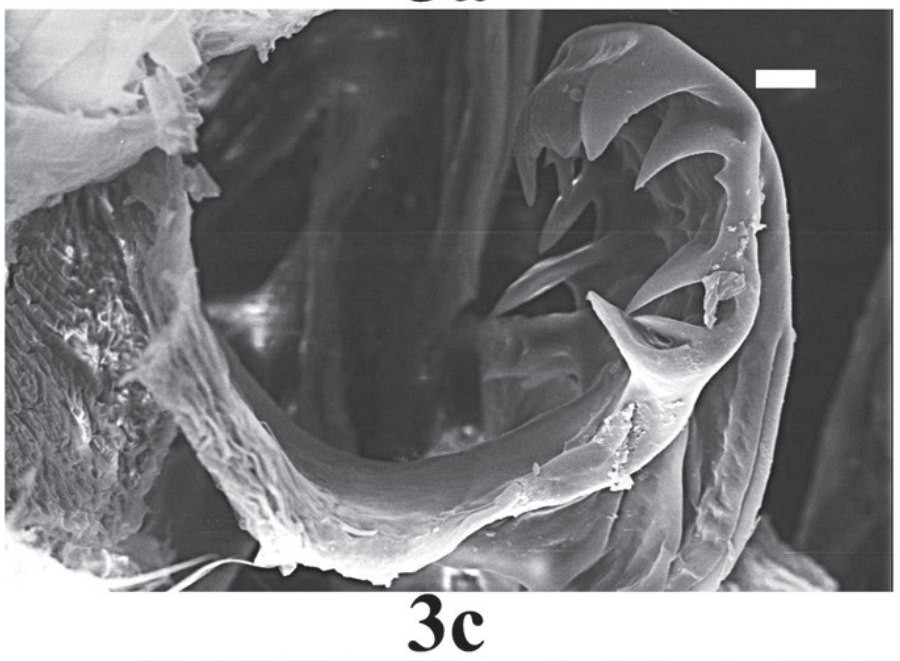

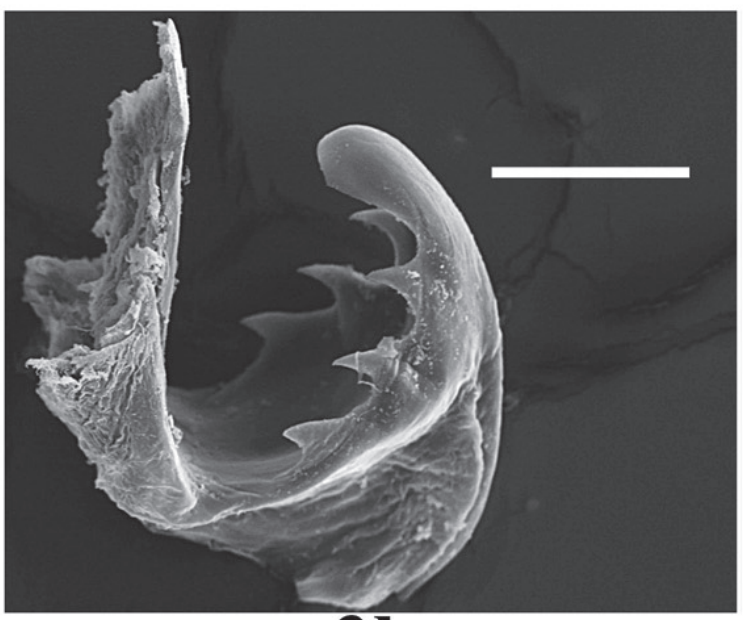

$3 \mathbf{b}$

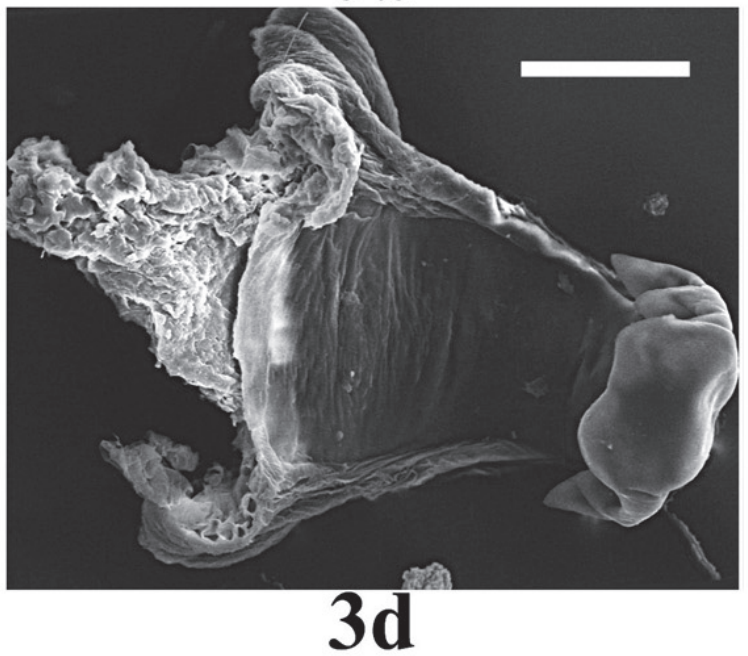

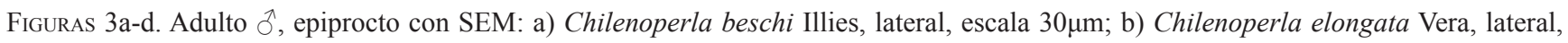
escala $100 \mu \mathrm{m}$; c) Ericiataperla puerilis Nov. comb, lateral, escala $20 \mu \mathrm{m}$; d) Dorsal, escala $100 \mu \mathrm{m}$.

FigurEs 3a-d. Adult $\widehat{\partial}$, epiproct with SEM: a) Chilenoperla beschi Illies, lateral, scale $30 \mu \mathrm{m}$; b) Chilenoperla elongata Vera, lateral, scale $100 \mu \mathrm{m}$; c) Ericiataperla puerilis Nov. comb, lateral, scale $20 \mu \mathrm{m}$; d) Dorsal, $100 \mu \mathrm{m}$. 


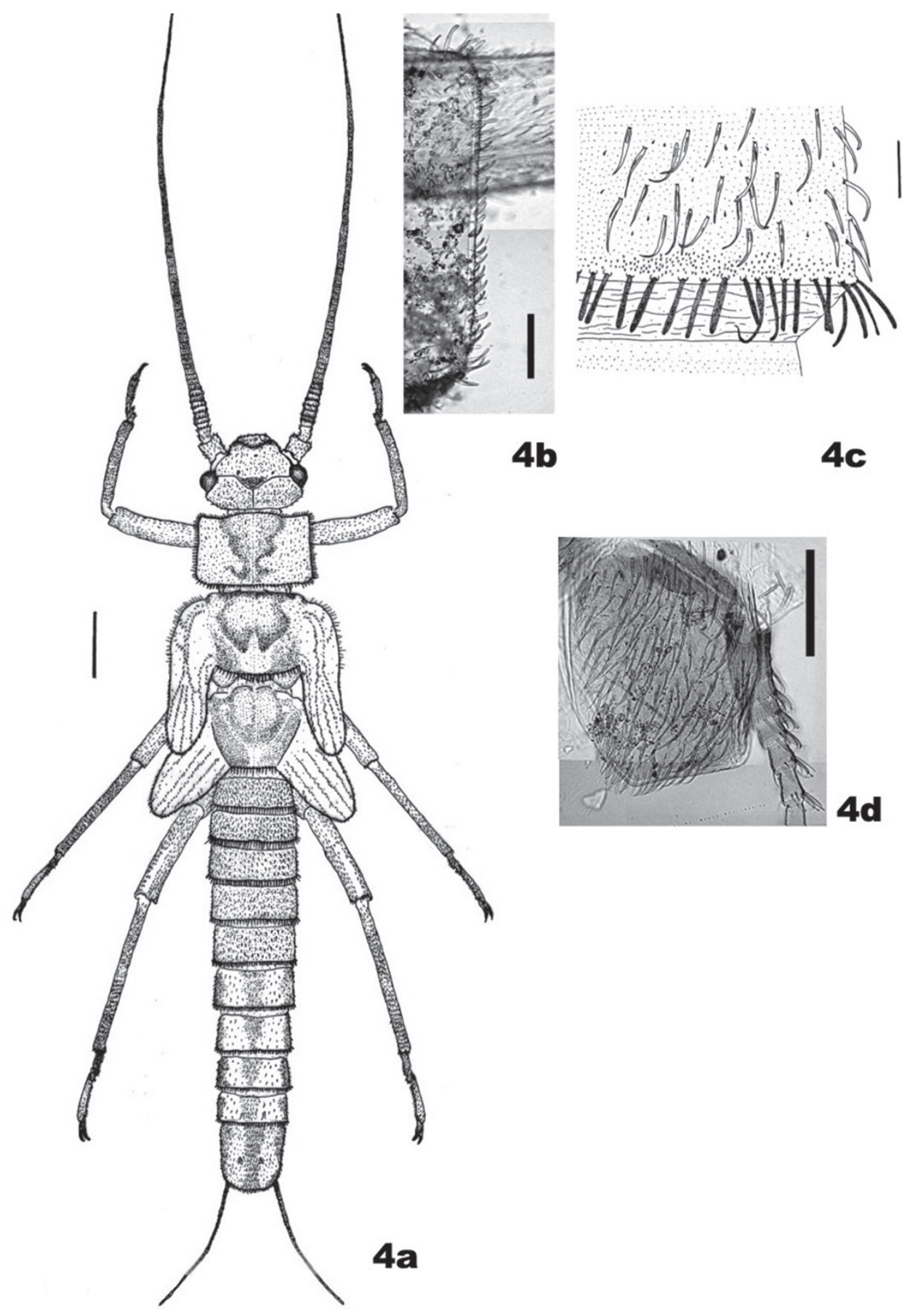

FIgURAS 4a-d. Ericiataperla puerilis Nov. comb. ninfa: a) Dorsal, escala 1,0mm; b) setas del margen lateral del pronoto, escala 0,5mm, c) Margen posterior del VII tergo abdominal, escala $0,1 \mathrm{~mm}$; d) Paraprocto, ventral, escala $0,25 \mathrm{~mm}$.

Figures 4a-d. Ericiataperla puerilis Nov. comb. nymph: a) Dorsal, scale $1.0 \mathrm{~mm}$; b) Setae on lateral margins of the pronotum, scale $0.5 \mathrm{~mm}$; c) posterior margins of the VII abdominal tergito, scale $0.1 \mathrm{~mm}$; d) Paraproct, ventral, scale $0.25 \mathrm{~mm}$. 
su cuatro distal curvado por sobre el esclerito central, con vesícula ventral desarrollada tras su primer tercio (Figs. 2 a y b), rama basal del paraprocto con lóbulo membranoso; falo esta compuesto por tres lóbulos, dos laterales, robustos y de forma piramidal, el central cilíndrico, se cubre parcialmente por los laterales. Cercos cortos de 7 a 8 segmentos robustos.

Imagos Hembra (Figs. 1b, c, e, f):

Dimensiones, longitudes en $\mathrm{mm}$ (promedios $\pm \mathrm{DE}$ ): cuerpo
$11,79 \pm 0,51$; antena $8,93 \pm 0,51$; cerco $0.64 \pm 0,20$; ala anterior $12,50 \pm 0,51$.

Hembras: Coloración corporal pardo anaranjado; Cabeza con oscurecimiento en el occipucio tras las ramas laterales de la sutura coronal; pronoto con impresiones oscuras muy contrastantes (Fig. 1e); Meso y meta noto pardo-oscuros, contrastantes con el resto del cuerpo; Patas con el tercio distal de los fémures y el cuarto basal de las tibias negro, el resto de la tibia es amarillo. Alas de lamina pardo claro.
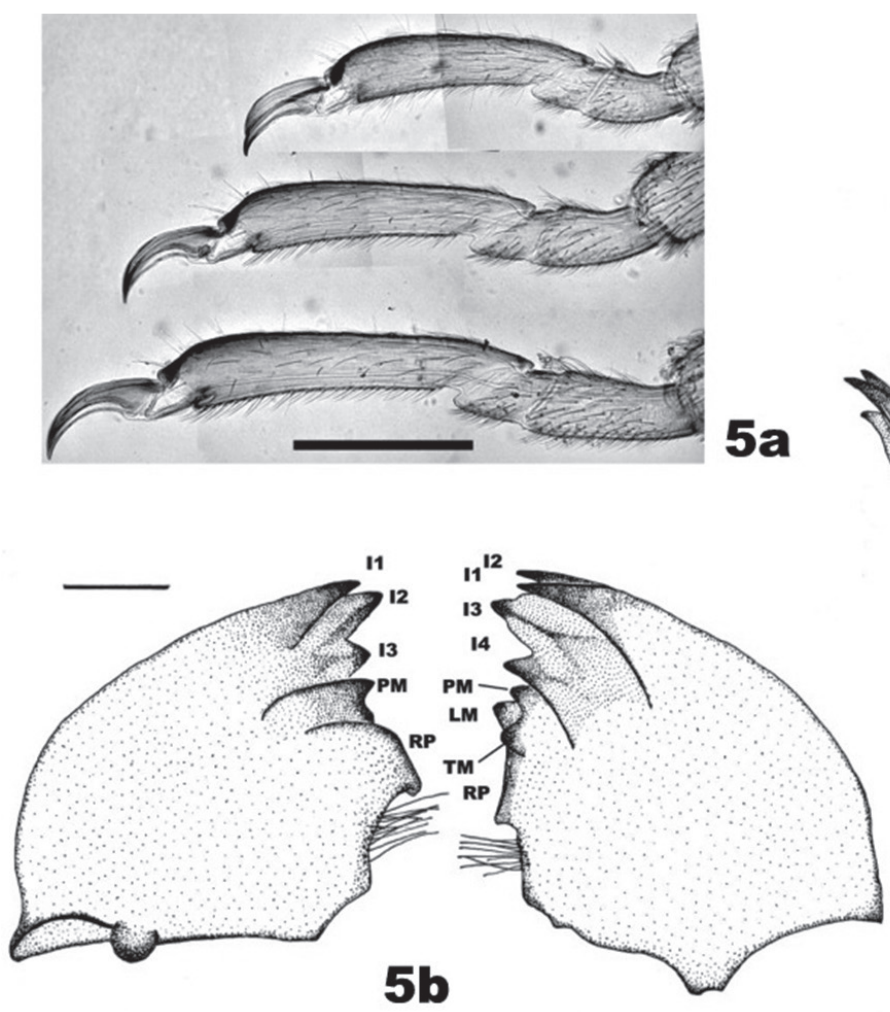

$5 a$
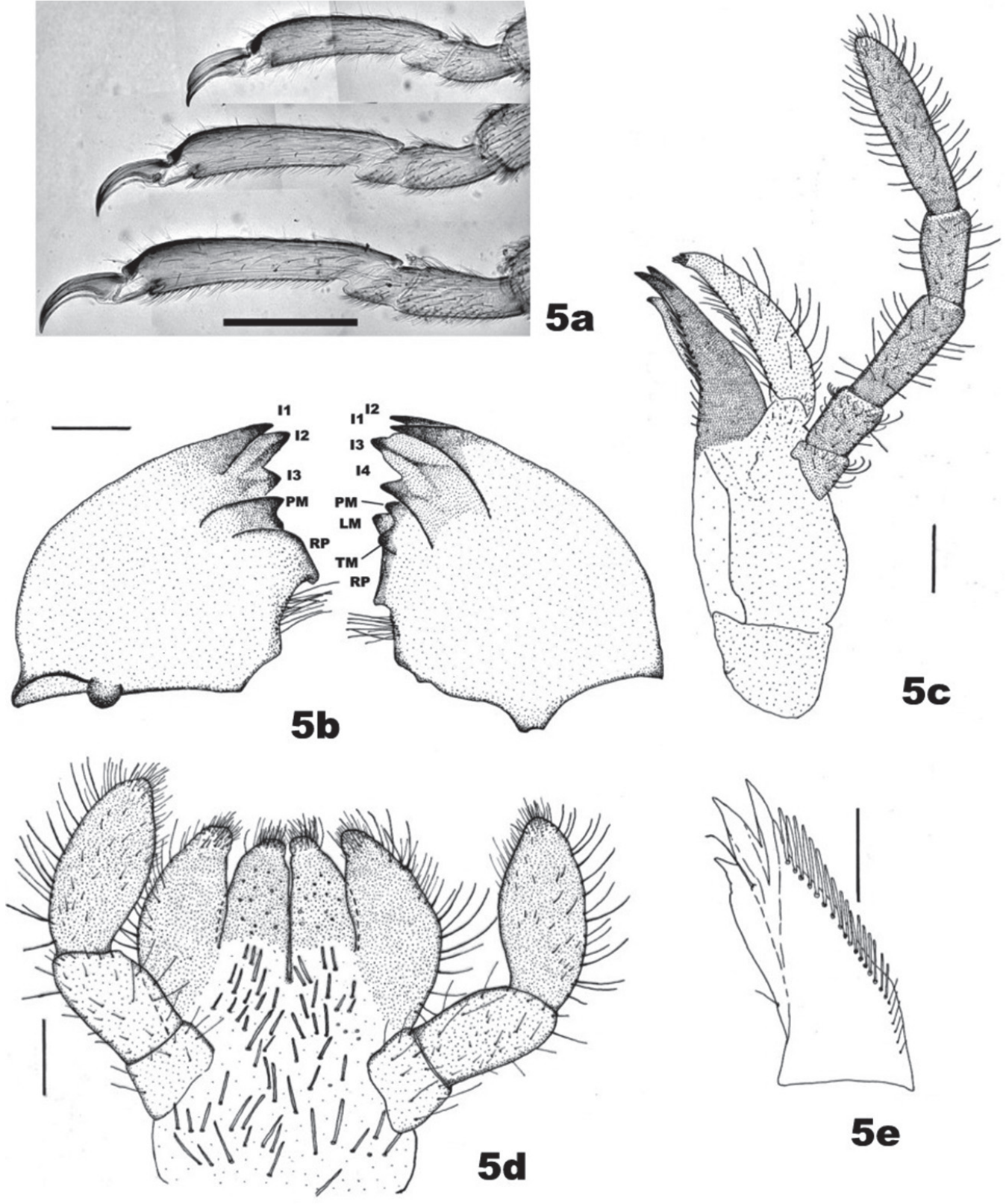

FIGURAS 5a-d. Ericiataperla puerilis Nov. comb. Ninfa: a) Tarsos de los tres pares de patas, escala 0,5mm; b) Mandíbulas, ventral, escala 0,2 $\mathrm{mm}$; c) Maxila, dorsal, escala 0,2 $\mathrm{mm}$, d) Labio, ventral, escala 0,2 $\mathrm{mm}$, e) Lacinia medial, escala 0,1 $\mathrm{mm}$.

FIGURES 5a-d. Ericiataperla puerilis Nov. comb. nymph: a) Tarsus of the three pairs of legs, scale 0.5mm; b) Mandibles, ventral, scale $0.2 \mathrm{~mm}$; c) Maxila, dorsal, scale $0.2 \mathrm{~mm}$; d) Labium, ventral, scale $0.2 \mathrm{~mm}$; e) Lacinia, medial, scale $0.1 \mathrm{~mm}$. 


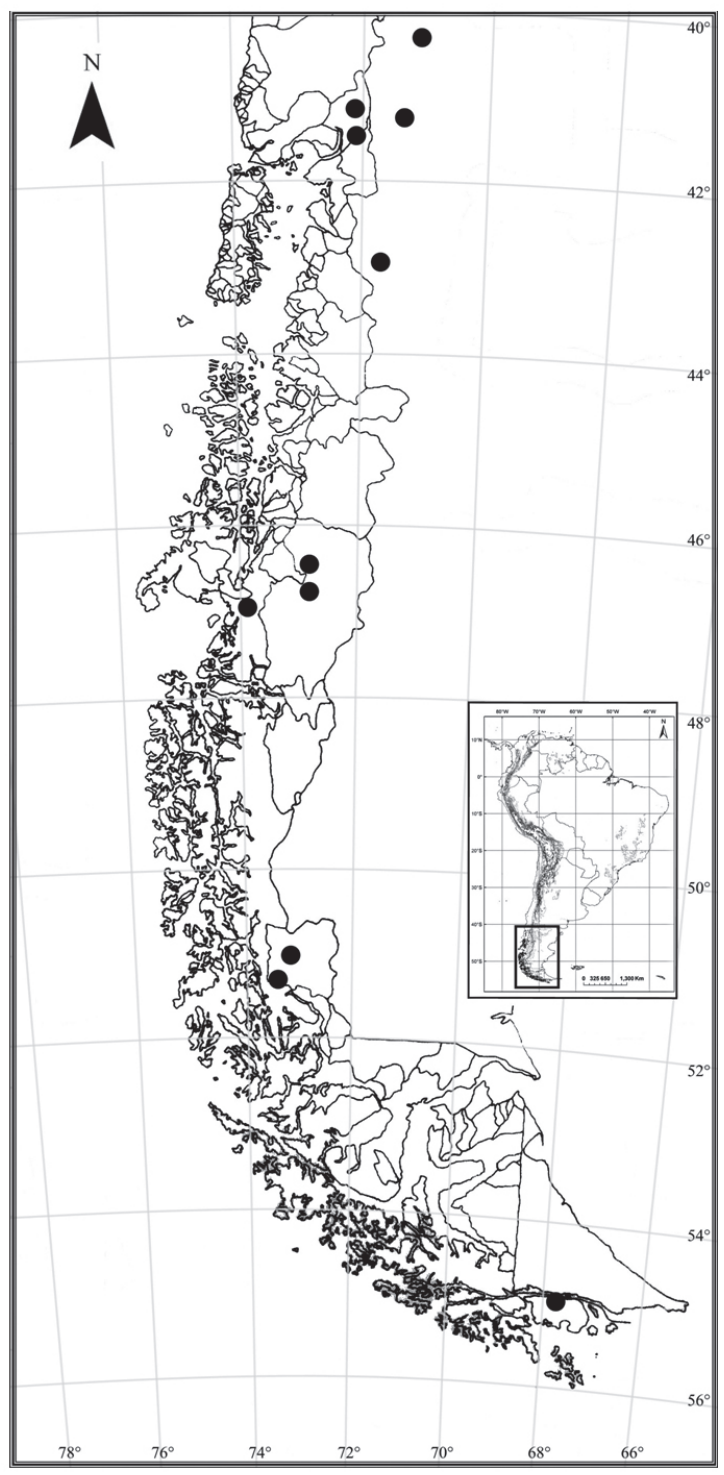

FIgURA 6. Distribución geográfica de Ericiataperla puerilis nov. comb. Se muestra un mapa de cuencas hidrográficas para Chile con las localidades conocidas para la especie.

FIGURE 6. Geographical distribution of Ericiataperla puerilis nov. comb. Hydrographic basins map for Chile is shown with known localities for the species.

La morfología es semejante a la de los machos, variando en la forma del tórax que es más angosto y de vértices un poco más redondeados (Fig. 1e). Abdomen con tergos I a IX no esclerosados; esternos mayoritariamente no esclerosados, Margen posterior de los segmentos V al VII con una tenue placa transversal de lados redondeados; Tergo VIII esclerosado latero-ventralmente, con su región media membranosa, amplia en el margen anterior y fuertemente estrechada hacia el margen posterior; margen posterior del tergo VIII prominente, cubre todo el segmento IX (Fig. 1f). Segmento IX esclerosado lateroventalmente. Segmento X esclerosado como anillo, en vista dorsal de margen posterior redondeado (Fig. 2c); con un par de profundas hendiduras medias acompañadas de un tubérculo liso y poco prominente a cada lado. Ceros de 8 segmentos robustos. Paraproctos subtriangulares.

REDESCRIPCIÓN DE LA NINFA DE ÚLTIMO ESTADÍO (Figs. 4 a-d; 5 a-e):

Coloración general pardo homogéneo, con algunas regiones más claras en tórax y abdomen, impresiones torácicas oscuras; bien esclerosadas; habitualmente con partículas de detrito en pleuras torácicas y abdomen; con evidentes cerdas cortas y rígidas en márgenes de tórax y abdomen.

Cabeza globosa (Fig. 4a); ramas anteriores de la sutura coronal doblada en la mitad, confluye perpendicularmente con el ojo; con una profunda depresión por delante de la sutura coronal; frente con ángulos anteriores prominentes, sutura frontoclipeal hendida medialmente, ocelos diminutos y equidistantes; clípeo y labro rectangulares y convexos; cabeza cubierta con abundantes cerdas cortas, oscuras y erizadas, clípeo y margen anterior de la frente con cerdas hialinas y curvas, ojos con escasas cerdas aovadas-agudas; antenas largas alcanzan el cuarto segmento abdominal, delgadas en el extremo distal, escapo y pedicelo cuadrados, con cerdas cortas, flagelo de 63 segmentos aproximadamente, cubiertos homogéneamente por cerdas cortas y rígidas, primeros siete o diez segmentos con cerdas de ápice curvo.

Armadura bucal: Mandíbulas (Fig. 5b) de forma globosa, con un mechón de cerdas por debajo de la región molar. Mandíbula derecha con incisivo apical (I1) y subapical (I2) agudos y paralelos, tercer incisivo (I3) grande y triangular; con un diente premolar (PM) agudo, ancho, plano, unido al tubérculo molar dorsal, que es agudo y prominente; región molar curvada dorsalmente, de superficie triangular. Mandíbula Izquierda con incisivos 1 y 2 subiguales, agudos, paralelos y separados solo distalmente, tercer incisivo prominente, agudo y de forma triangular, cuarto incisivo de forma triangular y agudo, solapado con el anterior; premolar pequeño y agudo, tubérculo molar prominente alineado con el premolar y el margen ventral de la muela, margen dorsal forma una lámina sobresaliente (LM), base de la muela protuberante. Hipofaringe monolobulada.

Maxilas (Fig. 5c) con palpos de 5 segmentos, según la proporción de longitudes 26:12:17:9:5; dos últimos segmentos ligeramente dilatados; con abundantes cerdas, rígidas, agudas y erizadas, sólo los dos primeros segmentos con cerdas curvas. Galea suavemente curvada hacia el ápice; ápice cubierto de cerdas planas; margen dorsal interno con 
una fila de cerdas largas y erizadas dispuestas por sobre la lacinia; cara externa con cerdas rígidas, agudas y erizadas. Lacinia muy esclerosada; de largo semejante al estipe; estrechada distalmente; con dos dientes apicales (Fig. 5e) planos, agudos y divergentes entre si; margen ventral con un diente subapical prominente, con una extensa lamina basal dilatada, lo que produce una cavidad medial (Fig. 5c y e); margen dorsal con una fila de cerdas, largas, rígidas, planas y de ápice agudo; con una cerda larga, de ápice agudo y curvado ubicada por delante del diente subapical; con una cerda, aguda y erizada, en el margen ventral por debajo del diente apical.

Labio (Fig. 5d) con palpos de 3 segmentos, el distal de largo semejante a la suma de los dos anteriores, según la proporción 10:7:3; los dos últimos segmentos del palpo dilatados medialmente; glosas y paraglosas anchas con cerdas apicales cortas, finas y abundantes; glosas con diminutas areolas dispersas sobre su cara ventral; paraglosas con cerdas largas y erizadas sólo en su cara dorsal; palpos y márgenes de las paraglosas con cerdas abundantes, rígidas, largas, agudas y curvadas anteriormente, en el último segmento del palpo las cerdas son más densas y cortas sobre su margen medio-distal.

Tórax: Pronoto (Fig. 4 a) transversal casi dos veces tan ancho como largo, de ancho semejante o mayor que la cabeza; de ángulos redondeados, lados paralelos y prominentes, margen anterior convexo en el centro y cóncavo antes de los ángulos, margen posterior convexo; superficie ligeramente convexa hacia los lados, sólo con algunas arrugas en las impresiones musculares; todo el borde presenta una fila de cerdas tipo púas (en forma de bastones cortos, cilíndricos, rígidos, oscuros, erizados, de ápice mayoritariamente redondeado, en algunas agudo) (Fig. 4b), superficie con igual tipo de cerdas.

Meso y metanoto (Fig. 4 a) convexos deprimidos en las impresiones musculares; margen posterior mesonotal convexo y metanotal recto; superficie con cerdas semejante al pronoto; márgenes posteriores con una fila de cerdas tipo púas; estuches alares bien desarrollados, con filas de cerdas sobre la venación y el margen; margen y ángulos anteriores del primer par con cerdas tipo púas; pro, meso y metapleuras con cerdas hialinas delgadas y flexibles (pueden atrapar detrito).

Patas delgadas, especialmente las tibias, que además son de mayor longitud hacia posterior; cubiertas de cerdas diminutas; tarsos según la siguiente proporción: primer par 13:2:4, segundo par 15:2:6 y tercer par 17:3:9; garras delgadas y extendidas (Fig. 5a).

Abdomen cilíndrico; Segmentos I-III divididos con pleuras laterales, el resto fundidos formando anillos; cada segmento cubierto de cerdas dispersas, gruesas y oscuras; margen posterior con una línea de cerdas tipo púas (Fig. 4c); con gran amplificación se observa una distribución dispersa de células cloridas por todo el segmento, hacia el margen posterior se desarrolla una banda de espinas diminutas interrumpidas en la zona media; desde el VI al X segmento las regiones laterales se aclaran generando una banda medial oscura (Fig. 4 a); X Tergo cubierto densamente por cerdas semejantes a las de los segmentos anteriores, pero más largas y de ápice agudo y curvado; con un par de verrugas circulares en la cara dorsal y ventral; apófisis anteriores cilíndricas, separadas por una mayor distancia que sus largos. Paraproctos (Fig. 4d) anchos, subtriangulares, en machos con un pequeño lóbulo dorsal despigmentado. Cercos lineares, tan largos como los últimos cuatro segmentos; con 22 segmentos aproximadamente, cubiertos de cerdas diminutas. Ramillete de branquias retráctil de color pardo.

\section{DiSTRIBUCIÓN GEOGRÁFICA Y HÁBITAT}

Los escasos datos disponibles ubican a la especie asociada a la cordillera de los Andes entre los 40 a 55 grados de latitud sur (Fig. 6), con grandes discontinuidades a falta de muestreos. Habita tanto en ríos como en esteros, varios parámetros ecológicos le han sido asociados en la literatura (Miserendino 2000, 2001, 2006; Miserendino \& Pizzolón 2000, 2003; Velásquez y Miserendino 2003a, 2003b, Figueroa et al. 2003).

\section{DISCUSIÓN}

La nueva combinación presenta variados rasgos morfológicos en oposición con el género Chilenoperla que sostienen la actual propuesta. En ambos imagos: 1.- forma del pronoto, notablemente transversal, de lados paralelos y en machos de vértices angulosos; 2.- nervadura alar sin o con escasas venas transversales interrumpidas, 3.- ausencia de celda subapical tras la horquilla de RS. En la genitalia masculina: 4.- morfología del epiprocto, con forma de "pala", ápice entero no prominente, 5.- dientes marginales transversales y de ápice poco agudo; 6.- paraproctos de cuarto apical doblado por sobre el tergo X. En las ninfas: 7.- cuerpo esclerosado; 8.- antenas sin cerdas modificadas; 9.- mandíbula izquierda con 4 incisivos; 10.- palpo maxilar largo, al menos 2,5 veces la lacinia; 11.- lacinia con dientes agudos de margen filoso; 12.- lacinia con lámina ventral; 13.- palpo labial con segmento distal dilatado medialmente; 14.- márgenes de patas, tórax y abdomen sin cerdas largas y cintiformes; 15.- pronoto transversal, con cerdas tipo "púas" en todo su margen; 16.- meso y metanoto con cerdas tipo "púas" en su margen posterior y margen lateral de los estuches alares; 17.- margen posterior de los segmentos abdominales con cerdas tipo "púas". 
En la genitalia Chilenoperla, las diferentes especies presentan epiproctos diagnósticos que varían en número y forma de las denticulaciones dorsales (Figs. 3 a y b), sin embargo estas son siempre marginales y la forma del epiprocto es aguzada hacia el ápice. Mientras que en Ericiataperla, la denticulación es transversal (Fig. 3c), donde cada diente se extiende como una lámina desde medial a marginal, el ápice del epiprocto es redondeado y en algunos casos de aspecto bilobulado (Fig. 3d). En cuanto a la nervadura alar, todas las especies de Chilenoperla presentan en ambos pares de alas una celda subapical tras la horquilla de RS, Illies (1963) describe como propias del género la presencia de abundantes venas transversales-interrumpidas, estas últimas no están presentes en Chilenoperla puelche como fue discutido por Vera (2012). Ambos caracteres no están presentes en Ericiataperla. En cuanto a la forma del pronoto, en todas las especies de Chilenoperla es cuadrado y de vértices claramente redondeados, mientras que en Ericiataperla se presenta transversal y de vértices angulosos, siendo en los machos más marcado en los vértices anteriores.

Ericiataperla puerilis presenta algunas semejanzas con otros géneros como es su patrón de nervadura alar próximo al de Antarctoperla y la rama basal del paraprocto con un lóbulo membranoso, semejante al de Neopentura semifusca, pero menos prominente. Zwick (1973) establece la sinonimia de Antarctoperla andersoni (Enderlein) indicando que las hembras descritas corresponderían a hembras del género Chilenoperla, de acuerdo a su descripción estas podrían corresponder a hembras de E. puerilis, quienes comparten su austral distribución y presentan semejanza en la venación alar con Antarctoperla.

En cuanto a las ninfas, la notable discrepancia entre Ericiataperla puerilis y Chilenoperla elongata fue anteriormente discutida por Vera (2008) y confirmadas con la descripción de C. puelche Vera (2012), así como la semejan semejanza de estas últimas con Pelurgoperla personata. La presunción de un nuevo taxa para $C$. puerilis fue comunicado de forma personal al Dr. Claudio Gilberto Froehlich, quien incorporo esta diferenciación en su clave para ninfas publicada en 2009. El número de especies de Chilenoperla puede incrementarse y aún permanecen desconocidas las ninfas de varias especies, con la información disponible se han identificado 11 rasgos que diferencian las ninfas de Ericiataperla y Chilenoperla.

En cuanto a la distribución geográfica de Ericiataperla, los escasos datos disponibles la ubican en el extremo Austral de Los Andes y Tierra del Fuego, mientras que las especies de Chilenoperla lo hacen en Chile Central y Sur, ambos se superponen entre los 40 y 45 grados de latitud Sur.
Finalmente esta contribución ayuda ha resolver las discrepancias al interior de Chilenoperla, lo que permitirá posteriormente redefinirlo y resolver su relación con el género Pelurgoperla.

\section{AGRADECIMIENTOS}

Al Museo Nacional de Historia Natural de Santiago, sección Entomología, por facilitar sus laboratorios y equipos. A quienes me han facilitado especímenes o ayuda para conseguirlos: Dr. Thibault Datry, Dr. Chistopher Anderson, Dra. Ma. Laura Miserendino, Dr. Pablo Pessacq, Álvaro Zúñiga y José Mondaca. Al Dr. Peter Zwick por el préstamo del material tipo de las especies de Chilenoperla desde la Colección Illies.

\section{BILIOGRAFÍA}

BÉTHoux, O. 2005. Wing venation pattern of Plecoptera (Insecta: Neoptera). Illiesia, 1(9):54-81.

Camousseight, A. \& Fontaine, J. 1990. The biological cycle of Baetis pentaphlebodes, Ujhelyi 1966, in a old meander of the Rhone River, France (Ephemeroptera: Baetidae). En: Mayflies and Stoneflies: Life story and Biology (Ed. Campbell, I.C.), pp.27-34. Kluwer Academic Publishers, Dordrecht,

Figueroa, R., C. Valdovinos, E. Araya \& Parra, O. 2003. Macroinvertebrados bentónicos como indicadores de calidad de agua de ríos del sur de Chile. Revista Chilena de Historia Natural 76(2):275-285.

Froehlich, C.G. 2009. Plecoptera. En: Microinvertebrados bentónicos sudamericanos. Sistemática y biología (Eds. Domínguez, E. \& H.R. Fernández), pp. 145-165. Fundacion Miguel Lillo, Tucumán, Argentina.

Froehlich, C.G. 2010. Catalogue of Neotropical Plecoptera. Illiesia, 6(12):118-205.

ILLIES, J. 1963. Revision des südamerikanischen Gripopterygidae (Plecoptera). Mitteilungen der Schweitzerischen Entomologischen Gesellschaft, 36(3):145-248.

McLellan, I. \& P. Zwick. 2007. New species of and keys to South American Gripopterygidae (Plecoptera). Illiesia 3(4):20-42.

Miserendino, M.L. \& Pizzolón, L.A. 2000. Macroinvertebrates of a fluvial system in Patagonia: altitudinal zonation and functional structure. Archiv fur Hydrobiology 150:55-83.

Miserendino, M.L. \& Pizzolón, L.A. 2003. Distribution of macroinvertebrate assemblages in the Azul-Quemquemtreu river basin, Patagonia, Argentina. New Zealand Journal of Marine and Freshwater Research 23(3):525-539

Miserendino, M.L. 2000. Distribución y variación estacional de Plecoptera en un sistema fluvial de cordillera Patagónica. Revista de la Sociedad Entomológica Argentina 59:149-60

Miserendino, M.L. 2001. Macroinvertebrate assemblages in Andean Patagonian rivers and streams. Hydrobiology 444:147-158.

Miserendino, M.L. 2006. Seasonal and spatial distribution of 
stoneflies in the Chubut River (Patagonia, Argentina). Hydrobiology 568:263-274.

Nelson, CH. 1973. Synopsis of the genus Chilenoperla (Plecoptera: Gripopterygidae). The Pan-Pacific Entomologist, 49(4):315-324.

Stark, BP., C.G. Froehlich \& Zuñiga, M. del C. 2009. South American Stoneflies (Plecoptera). En: Aquatic Biodiversity of Latin America (ABLA) (Eds. Adis, J., Arias, J.R., Golovatch, S., Wantzen, K.M. \& Rueda-Delgado), Vol 5. Pensolf, Sofia-Moscow, 154 pag.

Velásquez, S.M. \& Miserendino, M.L. 2003a. Análisis de la materia orgánica alóctona y organización funcional de macroinvertebrados en relación con el tipo de hábitat en ríos de montaña de Patagonia. Ecología Austral 13:67-82.

Velásquez, S.M. \& Miserendino, M.L. 2003b. Habitat type and macroinvertebrate assemblages in low order Patagonian streams. Archiv fur Hydrobiologie 158(4):461-483.

VerA, A. 2012. A new species of Chilenoperla (Plecoptera: Gripopterygidae) from the Andes of South America. Zootaxa 3268:63-68.

Vera, A. 2008. Una nueva especie de Chilenoperla (Plecoptera: Gripopterygidae) y las consecuencias taxonómicas del descubrimiento de su ninfa. Gayana 72(2):144-156.

Zwick, P. 1973. Die Plecopteren-Arten Enderleins (Insecta); Revision der Typen. Annales Zoologici.30 (16):471-507.

Recibido: 17.06 .14

Aceptado: 01.04.16 